\title{
Cystatin C level is associated with the recovery of renal function in cancer patients after onset of acute kidney injury
}

\author{
Rui Li, Xia Wang, Xiaoqian Zhao, Xiaohong Zhang, Honghai Chen, Yue Ma, Yandong Liu \\ Laboratory of the Fifth People's Hospital of Shenyang, Shenyang Key Laboratory of Cancer, Shenyang, China \\ Contributions: (I) Conception and design: R Li, Y Liu; (II) Administrative support: X Wang, X Zhao; (III) Provision of study materials or patients: X \\ Zhang, H Chen, Y Ma; (IV) Collection and assembly of data: R Li, X Wang, X Zhao, X Zhang; (V) Data analysis and interpretation: R Li, Y Liu; (VI) \\ Manuscript writing: All authors; (VII) Final approval of manuscript: All authors. \\ Correspondence to: Yandong Liu. Laboratory of the Fifth People's Hospital of Shenyang, Shenyang Key Laboratory of Cancer, Shenyang 110023 , \\ China. Email: Sylr2011@126.com.
}

Background The risk of injury to the kidney can be significantly exacerbated by the presence of tumors
and the effects of related treatments. Kidney injury associated with cancer is common in multiple myeloma,
tumor lysis syndrome, hematopoietic stem cell therapy, and chemotherapy. Cancer patients are at increased
risk of infection, sepsis, tumor lysis syndrome, drug-related toxicity, and other comorbidities, leading to a
significantly increased risk of acute kidney injury (AKI). This study retrospectively analyzed the clinical data
of AKI in cancer patients and explored the predictive value of Cystatin C (CysC) in the prognosis of cancer
patients with AKI.

Methods: Cancer patients attending the Fifth People's Hospital of Shenyang from April 2014 to March 2019 were enrolled according to inclusion and exclusion criteria. Cancer patients with AKI were divided into two groups according to the changes in renal function during the follow-up period: a renal function recovery group and a nonrecovery group. The differences in baseline data of the two groups were compared. Logistic univariate and multivariate regression analyses were conducted to determine the risk of renal function failure.

Results: A total of 3,127 cases were included. Among them, 659 cases (21.1\%) had AKI, and 2,468 cases had no AKI. Among the 659 AKI patients, 473 (71.8\%) patients' renal function recovered, while $186(28.2 \%)$ did not. Logistic univariate and multivariate regression analyses indicated that age [odds ratio $(\mathrm{OR})=1.133$, 95\% confidence interval (CI): 1.064-1.219], diabetes (OR =1.226, 95\% CI: 1.093-1.385), chronic kidney disease $(\mathrm{CKD})(\mathrm{OR}=1.347,95 \% \mathrm{CI}: 1.108-1.624)$, hematological malignancies $(\mathrm{OR}=1.174,95 \% \mathrm{CI}$ : 1.063-1.311), chemotherapy (OR =1.119, 95\% CI: 1.055-1.304), systolic blood pressure (OR =1.108, 95\% CI: $1.062-1.267)$, serum creatinine $(\mathrm{Scr})(\mathrm{OR}=1.262$, 95\% CI: $1.105-1.446)$, and CysC $(\mathrm{OR}=1.416,95 \%$ CI: 1.251-1.739) were related to the failure of renal function to recover after AKI.

Conclusions: Baseline $\mathrm{CysC}$ level is associated with the occurrence of $\mathrm{AKI}$ in cancer patients and a failure to recover renal function during follow-up.

Keywords: Cystatin C (CysC); cancer; acute kidney injury (AKI)

Submitted Nov 26, 2020. Accepted for publication Feb 04, 2021.

doi: $10.21037 /$ apm-21-191

View this article at: http://dx.doi.org/10.21037/apm-21-191

\section{Introduction}

The tremendous progress in cancer treatment has significantly improved the quality of life and survival rate of patients. However, the tumor itself and related treatments can significantly increase the risk of kidney injury. Kidney injury associated with cancer is common in multiple myeloma, tumor lysis syndrome, hematopoietic stem cell therapy, and chemotherapy. Cancer patients are at increased risk of infection, sepsis, tumor lysis syndrome, drug-related 
toxicity, and other comorbidities, leading to a significantly increased risk of acute kidney injury (AKI) $(1,2)$, which, in turn, further increases the mortality rate and may limit the progress or efficacy of related treatments $(1,2)$.

Two recent studies have shown that the total annual incidence of AKI in cancer patients is between $11 \%$ and $20 \%$, with the risk to hematological cancer patients being even higher $(3,4)$. A study in China surveyed 7 million patients, and the results showed that, depending on the type of hospital (community versus academic institution), the incidence of AKI (defined as a baseline increase in serum creatinine (Scr) by at least $50 \%$ ) ranged from $14 \%$ to $20 \%$ (5). Some studies have pointed out that the actual incidence of AKI is much higher $(60 \%)$ than that commonly reported $(6,7)$. Regarding the prognosis of AKI in cancer patients, one Danish cohort study found that $5 \%$ of patients required renal replacement therapy (RRT) within 1 year after the onset of AKI (3). Another study of highrisk populations showed that, depending on the severity of AKI and potential comorbidities, $8 \%$ to $60 \%$ of patients require RRT (8). More recent research investigating the incidence of AKI in 163,071 patients receiving systemic therapy (9) reported a total cumulative incidence of $9.3 \%$. The malignant tumors with the highest incidence of AKI in the past 5 years are myeloma $(26.0 \%)$, bladder cancer (19.0\%), and leukemia (15.4\%). Interestingly, between 2007 and 2014, the annual incidence of AKI increased from 18 to 52 per 1,000 person-years (9). The risk factors associated with the occurrence of AKI in cancer patients are both cancer-specific and patient-specific, with hematological malignancies, elderly patients, and potential chronic kidney disease (CKD) being the largest baseline risk factors.

The occurrence of AKI in cancer patients can lead to increased mortality, longer hospital stays, and reduced cancer remission rates (7,10-15). However, studies have found that among cancer patients with AKI, renal function can be recovered in $82 \%$ of patients and be partially recovered in $12 \%$ of patients, with only $6 \%$ of patients requiring continuous RRT (11). Therefore, predicting the long-term risk of cancer patients after AKI has important clinical significance. At present, the treatment of AKI in cancer patients is mainly supportive treatment and alternative treatment. In terms of drugs, statins may be effective $(16,17)$. The kidney is the only organ that removes Cystatin $\mathrm{C}(\mathrm{CysC})$ from the blood. The molecular weight of $\mathrm{CysC}$ is low, and it can thus be freely filtered by the glomerulus and distributed on the cell surface. It is almost completely absorbed in the proximal convoluted tubule (18), and its serum concentration mainly depends on kidney function. Consequently, this indicator can reflect the glomerular filtration rate (GFR) at an early stage. This study retrospectively analyzed the relevant data of AKI in cancer patients, and explored the predictive value of $\mathrm{Cys} C$ in the prognosis of cancer patients with AKI. We present the following article in accordance with the STROBE reporting checklist (available at http://dx.doi.org/10.21037/ apm-21-191).

\section{Methods}

\section{Study population}

Patients who were treated at the Fifth People's Hospital of Shenyang from April 2014 to March 2019 were included. The inclusion criteria for participants were the following: (I) age $\geq 18$ years; (II) clear diagnosis of malignant tumor; (III) life expectancy $\geq 3$ months; (IV) history of surgery, chemotherapy, or radiotherapy; (V) CysC test results available in the baseline data; (VI) regular renal function testing during the process of tumor treatment; (VII) fulfilling the definition of the AKI. Meanwhile, the exclusion criteria for participants were the following: (I) malignant tumors of the urinary system; (II) with severe heart failure, respiratory failure, liver failure; (III) with active connective tissue disease; (IV) incomplete followup data. This study was approved by the Ethics Committee of the Fifth People's Hospital of Shenyang. All procedures performed in this study involving human participants were in accordance with the Declaration of Helsinki (as revised in 2013). Individual consent for this retrospective analysis was waived.

\section{Data collection}

The clinical data of patients, including demographic data (sex, age, etc.), general data (height, weight, smoking history, etc.), basic medical history data (underlying diseases, baseline blood pressure, heart rate, routine blood test indicators, such as white blood cell count, hemoglobin concentration, liver function, renal function, electrolytes, blood lipids, blood glucose, apolipoprotein, CysC, etc.), tumor-related data (tumor location, clinical stage, treatment methods, related drugs and their dosage), and followup data (outcome, changes in renal function, etc.), were collected. The definition of AKI is judged and staged according to an elevated level of Scr and/or urine output: 
that is, (I) an increase in Scr within 48 hours exceeding $26.5 \mu \mathrm{mol} / \mathrm{L}$, or an increase in Scr exceeding 1.5 times the baseline, either confirmed or speculated to occur within 7 days; or (II) urine output $<0.5 \mathrm{~mL} / \mathrm{kg} \cdot \mathrm{h}$ lasting for more than 6 hours (AKI can be diagnosed if one of the above conditions is met) (19).

\section{Grouping}

According to whether or not the renal function returned to the baseline level during the 1-year follow-up period after the occurrence of AKI, the patients were divided into two groups: a recovery group and a nonrecovery group. The baseline of the two groups was compared, and differences in clinical data and outcomes during follow-up were analyzed.

\section{Statistical analysis}

Statistical processing was performed using SPSS 23.0 statistical software (IBM Corp., Armonk, NY, USA). Quantitative data were tested for normal homogeneity. If the data conform to a normal distribution are represented by mean \pm standard deviation, comparisons between groups were processed by the $t$-test. If the data did not conform to a normal distribution are represented by medians, the comparison between groups was processed by the ranksum test. The qualitative data are expressed by numbers and percentages, and comparison between groups was performed by $\chi^{2}$ test or Fisher's exact test if theoretical number was less than 1 . Logistic single factor and multivariate analyses of factors related to whether or not AKI could be resolved were also performed. A $\mathrm{P}$ value $<0.05$ indicated that the difference was statistically significant.

\section{Results}

\section{Patients baseline characteristics}

A total of 4,741 adult cancer patients (those with urinary system tumors were excluded) were diagnosed and treated in our hospital from April 2014 to March 2019, including 1,628 cases of lung cancer, 994 cases of gastric cancer, 847 cases of colorectal cancer, 516 cases of liver cancer, 325 cases of hematological malignancies, and 431 cases with other malignant tumors. According to the inclusion and exclusion criteria, 3,127 cases were ultimately included. Among them, 659 cases (21.1\%) had AKI, and 2,468 cases had no AKI. The patients' baseline information is shown in
Table 1.

\section{Follow-up outcome in cancer patients with AKI}

We further analyzed the follow-up data of cancer patients with AKI, and the results showed that during the 1-year follow-up, 473 patients (accounting for $71.8 \%$ of patients with AKI) recovered to the baseline level, and 186 patients (accounting for $28.2 \%$ of patients with AKI) did not. Comparison of baseline data between the two groups is shown in Table 2. We found that there were statistical differences in some indicators between patients who recovered renal function and those who did not, including in age, body mass index (BMI), blood pressure level, blood sugar level, tumor stage, diuretic use, $\mathrm{Cys} C$ and its change at the time of diagnosis of AKI, and others; however, the baseline level of creatinine, the increase in creatinine at the time of diagnosis of AKI, and the estimated glomerular filtration rate (eGFR) calculated from creatinine were not statistically different between the two groups.

\section{Factors associated with prognosis after the diagnosis of AKI in cancer patients}

Logistic univariate and multivariate regression analyses indicated that the baseline level of $\mathrm{Cys} C$ was closely correlated with the recovery of renal function in cancer patients after the occurrence of AKI. A high baseline level of CysC indicated a significantly higher risk of renal function failure. Univariate analysis revealed that the factors related to the failure of renal function recovery included age, BMI, hypertension, diabetes, CKD, hematological malignancies, chemotherapy, systolic blood pressure, hemoglobin, Scr, $\mathrm{Cys} \mathrm{C}$, and other factors. Further multivariate analysis suggested that age, diabetes, CKD, hematological malignancies, chemotherapy, systolic blood pressure, Scr, and $\mathrm{Cys} C$ were related to the failure of renal function to recover after AKI (see Table 3).

\section{Discussion}

This study retrospectively analyzed the clinical data of cancer patients with AKI in our hospital in recent years, and found that AKI is common in cancer patients. After AKI, some patients required RRT and experienced improved renal function after treatment but did not return to the baseline level. This significantly hampered the subsequent treatment of the patient's cancer, resulting in a significantly 
Table 1 Baseline data of patients

\begin{tabular}{|c|c|c|c|c|}
\hline Characteristics & AKI $(n=659)$ & Non-AKI $(n=2,468)$ & $t / \chi^{2}$ value & $P$ value \\
\hline Male (n, \%) & $436(66.2)$ & $1,547(62.7)$ & 2.713 & 0.100 \\
\hline Body mass index $\left(\mathrm{kg} / \mathrm{m}^{2}\right)$ & $22.7 \pm 2.1$ & $22.8 \pm 1.6$ & 1.328 & 0.184 \\
\hline Smoking (n, \%) & $87(13.2)$ & 319 (12.9) & 0.035 & 0.851 \\
\hline Hypertension (n, \%) & $62(9.4)$ & 171(6.9) & 4.637 & 0.031 \\
\hline Diabetes (n, \%) & $43(6.5)$ & $91(3.7)$ & 10.212 & 0.001 \\
\hline Chronic kidney disease (n, \%) & $75(11.4)$ & $220(8.9)$ & 3.704 & 0.054 \\
\hline \multicolumn{5}{|l|}{ Tumor } \\
\hline Liver cancer (n, \%) & $68(10.3)$ & $273(11.1)$ & 0.230 & 0.587 \\
\hline Blood cancer (n, \%) & $69(10.5)$ & $152(6.2)$ & 14.721 & 0.0001 \\
\hline Other cancer (n, \%) & $65(9.9)$ & $213(8.6)$ & 0.976 & 0.323 \\
\hline \multicolumn{5}{|l|}{ Therapy } \\
\hline Surgery (n, \%) & $482(73.1)$ & $1,827(74.0)$ & 0.212 & 0.646 \\
\hline Chemotherapy (n, \%) & $426(64.6)$ & $1,425(57.7)$ & 10.265 & 0.001 \\
\hline Radiotherapy (n, \%) & $218(33.1)$ & $833(33.8)$ & 0.105 & 0.746 \\
\hline Fast glucose (mmol/L) & $5.62 \pm 0.31$ & $5.64 \pm 0.29$ & 1.550 & 0.121 \\
\hline White blood cell $\left(\times 10^{9} / L\right)$ & $5.48 \pm 0.73$ & $5.53 \pm 0.62$ & 1.736 & 0.083 \\
\hline Neutrophil (×10\%/L) & $3.19 \pm 0.44$ & $3.23 \pm 0.33$ & 1.922 & 0.055 \\
\hline Red blood cell $\left(\times 10^{9} / \mathrm{L}\right)$ & $4.37 \pm 0.51$ & $4.39 \pm 0.45$ & 0.985 & 0.325 \\
\hline Hemoglobin (g/L) & $140.2 \pm 7.4$ & $140.5 \pm 7.2$ & 0.945 & 0.345 \\
\hline Platelet $\left(\times 10^{9} / \mathrm{L}\right)$ & $209.7 \pm 24.5$ & $211.2 \pm 23.8$ & 1.428 & 0.153 \\
\hline Creatine (mmol/L) & $79.3 \pm 8.5$ & $78.7 \pm 7.7$ & 1.728 & 0.082 \\
\hline Uric acid (mg/L) & $326.9 \pm 28.1$ & $324.5 \pm 26.3$ & 1.794 & 0.073 \\
\hline Alanine aminotransferase (U/L) & $23.8 \pm 3.1$ & $23.7 \pm 2.5$ & 0.865 & 0.387 \\
\hline Total cholesterol (mmol/L) & $4.72 \pm 0.43$ & $4.75 \pm 0.41$ & 1.651 & 0.099 \\
\hline Triglyceride (mmol/L) & $1.95 \pm 0.36$ & $1.93 \pm 0.33$ & 1.355 & 0.175 \\
\hline Cystatin C (mg/L) & $1.45 \pm 0.22$ & $1.32 \pm 0.18$ & 15.676 & $<0.001$ \\
\hline
\end{tabular}

$\mathrm{AKI}$, acute kidney injury. 
Table 2 Comparison of baseline data between the recovery group and the nonrecovery group

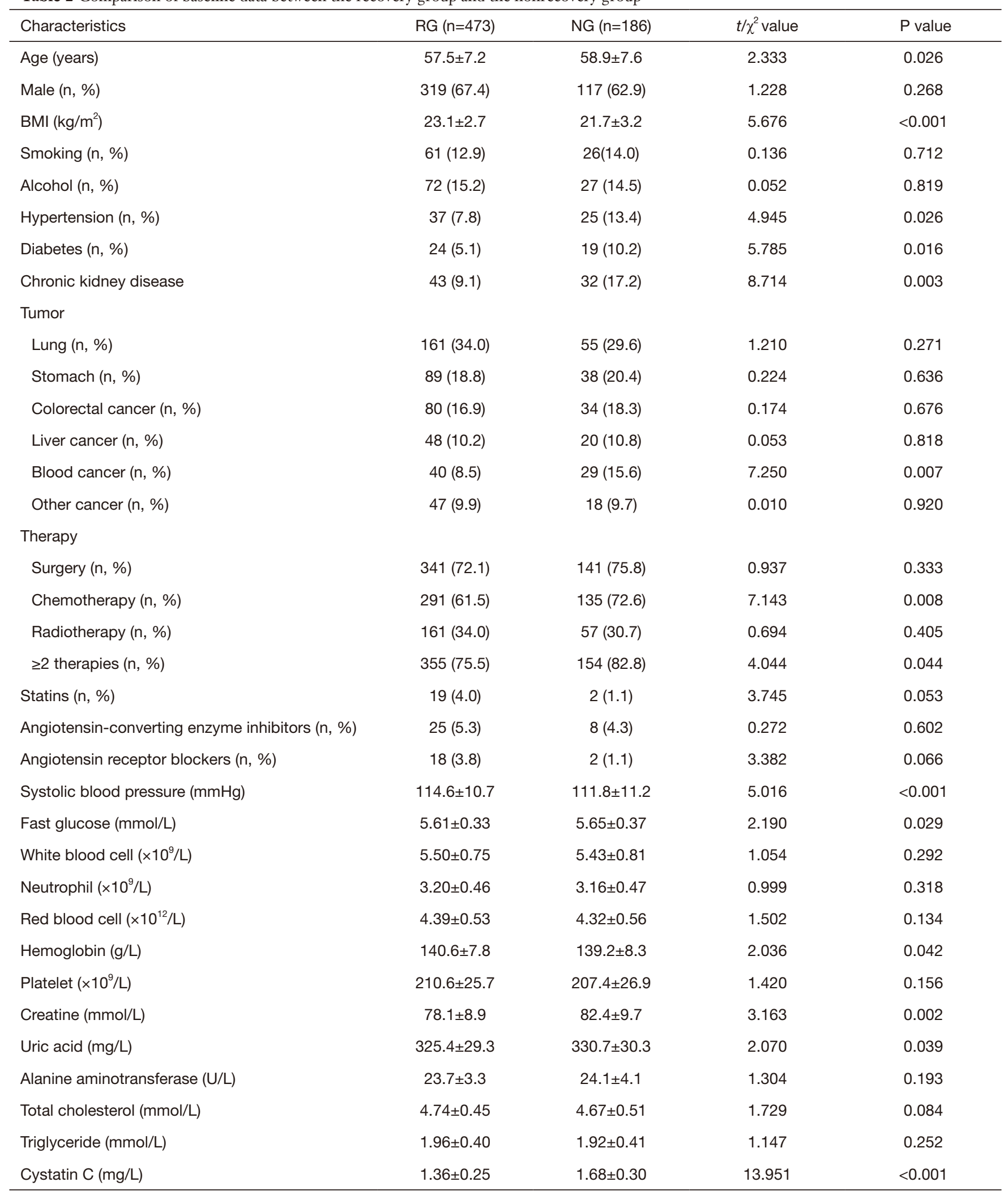

RG, recovery group; NG, nonrecovery group. 
Table 3 Multivariate analysis and factors that cause renal failure in cancer patients after AKI

\begin{tabular}{lccc}
\hline Factors & OR & 95\% Cl & P value \\
\hline Age & 1.133 & $1.064-1.219$ & 0.031 \\
Diabetes & 1.226 & $1.093-1.385$ & 0.026 \\
Chronic kidney disease & 1.347 & $1.108-1.624$ & 0.022 \\
Blood cancer & 1.174 & $1.063-1.311$ & 0.037 \\
Chemotherapy & 1.119 & $1.055-1.304$ & 0.041 \\
Systolic blood pressure & 1.108 & $1.062-1.267$ & 0.044 \\
Creatine & 1.262 & $1.105-1.446$ & 0.019 \\
CysC & 1.416 & $1.251-1.739$ & 0.012 \\
\hline
\end{tabular}

$\mathrm{AKI}$, acute kidney injury; OR, odds ratio; $\mathrm{Cl}$, confidence interval; CysC, Cystatin $\mathrm{C}$.

worse prognosis. However, some of these patients were able to recover baseline levels after treatment. Further analysis found that the patient's baseline level of treatment CysC was significantly related to whether or not the patient's renal function could return to the baseline level. Other risk factors related to the recovery of kidney function after AKI in cancer patients included age, diabetes, CKD, hematological malignancies, chemotherapy, systolic blood pressure, and Scr. Some previous studies have suggested that these factors are closely related to the risk of AKI in cancer patients (1-4). In our study, we further found that these factors were related to the recovery of renal function after the occurrence of AKI. The results of these studies were consistent with each other, suggesting that patients with this type of cancer have an increased risk of AKI and have an increased risk of renal function failure after AKI.

Cystatin C is a biomarker that has been studied over recent years. It is mainly used in the prediction of renal function and has also been widely discussed in other diseases. The level of $\mathrm{CysC}$ is independent of sex and size, and reaches adult levels within the first year after birth (20). The results of a meta-analysis study showed that $\mathrm{Cys} C$ has high diagnostic sensitivity for the detection of mild GFR decrease (21). Its role in the diagnosis and prediction of AKI has also been thoroughly examined $(22,23)$. Some researchers believe that $\mathrm{CysC}$ is an accurate biomarker for the early detection of AKI and may be superior to creatinine for some populations; however, the relevant findings are sometimes inconsistent. For important outcomes, such as death and RRT, CysC has also demonstrated good predictive value. More research is needed, and the focus should be on the cost-effectiveness of early detection of AKI with $\mathrm{Cys} C$ compared to that with creatinine, and whether or not the indicators have complementary value. As $\mathrm{Cys} C$ is produced by nucleated cells, increased tumor burden may lead to increased serum levels of $\mathrm{CysC}$ (24). This elevated level has been observed in patients with metastatic cancer, but in the absence of related technology, it is impossible to assess whether the reduced kidney function is due to the tumor burden or the disease and its treatment. Cystatin $\mathrm{C}$ has been shown to be widely expressed in cells and tissues of various human solid tumors-albeit in uneven distribution-especially in head and neck cancer (25), lung cancer (26), gastrointestinal tumors (27), ovarian cancer (28), prostate cancer (29), kidney cancer (30), breast cancer (31), melanoma (32), central nervous system (CNS) tumors (33), hematological malignancies (such as leukemia) (34), lymphoma (35), and myeloma (36). Interestingly, clinical studies have emphasized the significant association between changes in the expression level of this molecule and clinical outcomes in cancer patients $(37,38)$. Although these observations support the hypothesis that $\mathrm{Cys} C$ is involved in cancer, its specific role in malignant diseases is still unclear. In fact, studies specifically aimed at revealing the role of $\mathrm{Cys} C$ in tumor growth have shown that this molecule plays a series of complex roles that may inhibit or promote tumor cell growth and spread (39). Experimental studies conducted to clarify the potential opposite mechanism of action have shown that these phenomena may be partly related to the cathepsin inhibitory function of $\mathrm{CysC}$, and therefore, to the context-sensitive role of these enzymes in different types of cancers and diseases, or to the different steps of malignant progression $(39,40)$. However, emerging evidence suggests 
that in addition to cathepsin inhibition, other mechanisms unrelated to this function may also explain the complex effects of CysC on tumor cell growth and spread $(41,42)$.

Our study found that the baseline level of $\mathrm{CysC}$ can not only predict the occurrence of $\mathrm{AKI}$ in cancer patients, but is also related to the subsequent changes in renal function, independent of other risk factors. This result has important clinical significance. First, it implies that $\mathrm{Cys} C$ can predict the risk of AKI in cancer patients. In high-risk patients, if $\mathrm{Cys} C$ is high, it is unlikely that AKI will be resolved. These patients should be closely monitored and given intensive treatment. More specifically, clinicians can avoid drugs that damage kidney function, reduce the burden on the kidneys, administer drugs that protect kidney function, and perform other related measures. Though only about $18 \%$ patients cannot fully recover from cancer-related AKI, with the increasing absolute number and incidence of cancer cases, we believe CysC play an important role in predicting the prognosis of AKI in cancer patients.

Some limitations to this study should also be addressed. First, the study included a comparatively small number of patients and was single-center and retrospective in design; thus, the statistical power of the evidence is relatively weak. Second, the follow-up time of the study was short, and the relationship between subsequent changes in renal function and $\mathrm{Cys} C$ remains uncertain. Therefore, further multicenter, large-sample studies with longer follow-up times are needed.

\section{Acknowledgments}

Funding: None.

\section{Footnote}

Reporting Checklist: The authors have completed the STROBE reporting checklist. Available at http://dx.doi. org/10.21037/apm-21-191

Data Sharing Statement: Available at http://dx.doi. org/10.21037/apm-21-191

Conflicts of Interest: All authors have completed the ICMJE uniform disclosure form (available at http://dx.doi. org/10.21037/apm-21-191). The authors have no conflicts of interest to declare.

Ethical Statement: The authors are accountable for all aspects of the work in ensuring that questions related to the accuracy or integrity of any part of the work are appropriately investigated and resolved. This study was approved by the Ethics Committee of the Fifth People's Hospital of Shenyang. All procedures performed in this study involving human participants were in accordance with the Declaration of Helsinki (as revised in 2013). Individual consent for this retrospective analysis was waived.

Open Access Statement: This is an Open Access article distributed in accordance with the Creative Commons Attribution-NonCommercial-NoDerivs 4.0 International License (CC BY-NC-ND 4.0), which permits the noncommercial replication and distribution of the article with the strict proviso that no changes or edits are made and the original work is properly cited (including links to both the formal publication through the relevant DOI and the license). See: https://creativecommons.org/licenses/by-nc-nd/4.0/.

\section{References}

1. Lameire N, Vanholder R, Van Biesen W, et al. Acute kidney injury in critically ill cancer patients: an update. Crit Care 2016;20:209.

2. Rosner MH, Perazella MA. Acute Kidney Injury in Patients with Cancer. N Engl J Med 2017;376:1770-81.

3. Salahudeen AK, Doshi SM, Pawar T, et al. Incidence rate, clinical correlates, and outcomes of AKI in patients admitted to a comprehensive cancer center. Clin J Am Soc Nephrol 2013;8:347-54.

4. Christiansen CF, Johansen MB, Langeberg WJ, et al. Incidence of acute kidney injury in cancer patients: a Danish population-based cohort study. Eur J Intern Med 2011;22:399-406.

5. Jin J, Wang Y, Shen Q, et al. Acute kidney injury in cancer patients: A nationwide survey in China. Sci Rep 2019;9:3540.

6. Darmon M, Vincent F, Canet E, et al. Acute kidney injury in critically ill patients with haematological malignancies: results of a multicentre cohort study from the Groupe de Recherche en Réanimation Respiratoire en OncoHématologie. Nephrol Dial Transplant 2015;30:2006-13.

7. Lahoti A, Kantarjian H, Salahudeen AK, et al. Predictors and outcome of acute kidney injury in patients with acute myelogenous leukemia or high-risk myelodysplastic syndrome. Cancer 2010;116:4063-8.

8. Darmon M, Ciroldi M, Thiery G, et al. Clinical review: specific aspects of acute renal failure in cancer patients. 
Crit Care 2006;10:211.

9. Kitchlu A, McArthur E, Amir E, et al. Acute Kidney Injury in Patients Receiving Systemic Treatment for Cancer: A Population-Based Cohort Study. J Natl Cancer Inst 2019;111:727-36.

10. Canet E, Zafrani L, Lambert J, et al. Acute kidney injury in patients with newly diagnosed high-grade hematological malignancies: impact on remission and survival. PLoS One 2013;8:e55870.

11. Soares M, Salluh JI, Carvalho MS, et al. Prognosis of critically ill patients with cancer and acute renal dysfunction. J Clin Oncol 2006;24:4003-10.

12. Samuels J, Ng CS, Nates J, et al. Small increases in serum creatinine are associated with prolonged ICU stay and increased hospital mortality in critically ill patients with cancer. Support Care Cancer 2011;19:1527-32.

13. Maccariello E, Valente C, Nogueira L, et al. Outcomes of cancer and non-cancer patients with acute kidney injury and need of renal replacement therapy admitted to general intensive care units. Nephrol Dial Transplant 2011;26:537-43.

14. Park MR, Jeon K, Song JU, et al. Outcomes in critically ill patients with hematologic malignancies who received renal replacement therapy for acute kidney injury in an intensive care unit. J Crit Care 2011;26:107.e1-6.

15. Libório AB, Abreu KL, Silva GB Jr, et al. Predicting hospital mortality in critically ill cancer patients according to acute kidney injury severity. Oncology 2011;80:160-6.

16. Joshi SS, Ruth K, Smaldone MC, et al. Perioperative Statin Use and Acute Kidney Injury in Patients Undergoing Partial Nephrectomy. Kidney Cancer 2018;2:47-55.

17. James MT, Bhatt M, Pannu N, et al. Long-term outcomes of acute kidney injury and strategies for improved care. Nat Rev Nephrol 2020;16:193-205.

18. Aslan O, Demir M, Koseoglu M. Kidney injury molecule levels in type 2 diabetes mellitus. J Clin Lab Anal 2016;30:1031-6.

19. Khwaja A. KDIGO clinical practice guidelines for acute kidney injury. Nephron Clin Pract 2012;120:c179-84.

20. Finney H, Newman DJ, Thakkar H, et al. Reference ranges for plasma cystatin $\mathrm{C}$ and creatinine measurements in premature infants, neonates, and older children. Arch Dis Child 2000;82:71-5.

21. Dharnidharka VR, Kwon C, Stevens G. Serum cystatin $\mathrm{C}$ is superior to serum creatinine as a marker of kidney function: a meta-analysis. Am J Kidney Dis 2002;40:221-6.

22. Bagshaw SM, Bellomo R. Cystatin C in acute kidney injury. Curr Opin Crit Care 2010;16:533-9.
23. Choudhary A, Basu S, Dey SK, et al. Association and prognostic value of serum Cystatin C, IL-18 and Uric acid in urological patients with acute kidney injury. Clin Chim Acta 2018;482:144-8.

24. Kos J, Stabuc B, Cimerman N, et al. Serum cystatin C, a new marker of glomerular filtration rate, is increased during malignant progression. Clin Chem 1998;44:2556-7.

25. Yuan J, Xu M, Li J, et al. Prognostic value of cystatin $\mathrm{C}$ in patients with nasopharyngeal carcinoma: a retrospective study of 1063 patients. Clinics (Sao Paulo) 2016;71:338-43.

26. Hmmier A, O'Brien ME, Lynch V, et al. Proteomic analysis of bronchoalveolar lavage fluid (BALF) from lung cancer patients using label-free mass spectrometry. BBA Clin 2017;7:97-104.

27. Zinkin NT, Grall F, Bhaskar K, et al. Serum proteomics and biomarkers in hepatocellular carcinoma and chronic liver disease. Clin Cancer Res 2008;14:470-7.

28. Zhang W, Wang S, Wang Q, et al. Overexpression of cysteine cathepsin $\mathrm{L}$ is a marker of invasion and metastasis in ovarian cancer. Oncol Rep 2014;31:1334-42.

29. Larkin SE, Johnston HE, Jackson TR, et al. Detection of candidate biomarkers of prostate cancer progression in serum: a depletion-free 3D LC/MS quantitative proteomics pilot study. Br J Cancer 2016;115:1078-86.

30. Guo S, Xue Y, He Q, et al. Preoperative serum cystatin-C as a potential biomarker for prognosis of renal cell carcinoma. PLoS One 2017;12:e0178823.

31. Ziegler YS, Moresco JJ, Yates JR 3rd, et al. Integration of Breast Cancer Secretomes with Clinical Data Elucidates Potential Serum Markers for Disease Detection, Diagnosis, and Prognosis. PLoS One 2016;11:e0158296.

32. Matarrese P, Ascione B, Ciarlo L, et al. Cathepsin B inhibition interferes with metastatic potential of human melanoma: an in vitro and in vivo study. Mol Cancer 2010;9:207.

33. Lah TT, Nanni I, Trinkaus $M$, et al. Toward understanding recurrent meningioma: the potential role of lysosomal cysteine proteases and their inhibitors. J Neurosurg 2010;112:940-50.

34. Samaiya M, Bakhshi S, Shukla AA, et al. Epigenetic regulation of cathepsin $\mathrm{L}$ expression in chronic myeloid leukaemia. J Cell Mol Med 2011;15:2189-99.

35. Hammouda NE, Salah El-Din MA, El-Shishtawy MM, et al. Serum Cystatin C as a Biomarker in Diffuse Large B-Cell Lymphoma. Sci Pharm 2017;85:9.

36. Terpos E, Katodritou E, Tsiftsakis E, et al. Cystatin-C is an independent prognostic factor for survival in multiple 
myeloma and is reduced by bortezomib administration. Haematologica 2009;94:372-9.

37. Magister S, Kos J. Cystatins in immune system. J Cancer 2013;4:45-56.

38. Kos J, Werle B, Lah T, et al. Cysteine proteinases and their inhibitors in extracellular fluids: markers for diagnosis and prognosis in cancer. Int J Biol Markers 2000;15:84-9.

39. M.Prunk, M. Perišić Nanut, J. Sabotič, et al. Cystatins, cysteine peptidase inhibitors, as regulators of immune cell cytotoxicity, Period Biol 2016;118:353-62.

40. Kos J, Nanut MP, Prunk M, et al. Cystatin F as a regulator

Cite this article as: Li R, Wang X, Zhao X, Zhang X, Chen H, Ma Y, Liu Y. Cystatin C level is associated with the recovery of renal function in cancer patients after onset of acute kidney injury. Ann Palliat Med 2021;10(2):2158-2166. doi: 10.21037/ apm-21-191 of immune cell cytotoxicity. Cancer Immunol Immunother 2018;67:1931-8.

41. Tian M, Schiemann WP. Preclinical efficacy of cystatin C to target the oncogenic activity of transforming growth factor Beta in breast cancer. Transl Oncol 2009;2:174-83.

42. Beker BM, Corleto MG, Fieiras C, et al. Novel acute kidney injury biomarkers: their characteristics, utility and concerns. Int Urol Nephrol 2018;50:705-13.

(English Language Editor: J. Gray) 